\begin{tabular}{|c|c|}
\hline Title & Dispersion reduction in generation of high-order optical vortex using axially symmetric half-wave plates \\
\hline Author(s) & Sakamoto, Moritsugu; Fukumoto, Ryohei; Murakami, Naoshi; Morita, Ryuji; Oka, Kazuhiko \\
\hline Citation & $\begin{array}{l}\text { Optical Review, 22(1), 174-178 } \\
\text { https://doi.org/10.1007/s10043-015-0026-4 }\end{array}$ \\
\hline Issue Date & 2015-02-25 \\
\hline Doc URL & http:/hdl.handle.net/2115/64578 \\
\hline Rights & 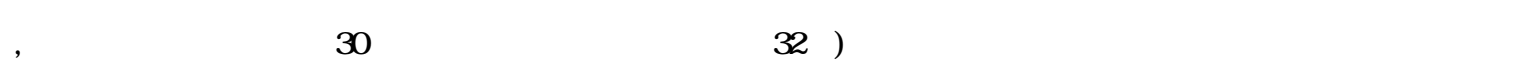 \\
\hline Type & article (author version) \\
\hline File Information & OR_22_174.pdf \\
\hline
\end{tabular}

Instructions for use 


\title{
Dispersion reduction in generation of high-order optical vortex using axially-symmetric half-wave plates
}

\author{
Moritsugu Sakamoto, ${ }^{1 *}$ Ryohei Fukumoto, ${ }^{1}$ Naoshi Murakami, ${ }^{2}$ Ryuji Morita, ${ }^{2,3}$ and Kazuhiko Oka ${ }^{2}$ \\ ${ }^{1}$ Division of Applied Physics, Graduate School of Engineering, Hokkaido University, Japan \\ ${ }^{2}$ Division of Applied Physics, Faculty of Engineering, Hokkaido University, Japan \\ ${ }^{3}$ JST, CREST, Japan
}

We present the topological-charge-dispersion reduction scheme in optical vortex generation using axially-symmetric half-wave plates (AHPs). The topological-charge dispersion caused by retardation errors of AHPs and other waveplates is decreased by eliminating unwanted circular-polarization components.

Keywords: Optical vortex, Singular optics, Broadband optical vortex, Polarization, Achromatic optics

Optical vortices (OVs) with broadband spectra have attractive applications such as ultrafast spectroscopy ${ }^{1)}$ and astronomical coronagraph. ${ }^{2)}$ For the generation of a broadband $\mathrm{OV}$, a scheme free from any type of dispersions is required, while most of the standard techniques for the OV generation, such as the methods using a spatial light modulator (SLM) or a spiral phase-plate (SPP), heavily depend on wavelength. Accordingly, the generation of a broadband OV using such a standard technique requires the compensation of the strong dispersions. ${ }^{3,4)}$ In contrast, the OV generation using axially symmetric half-wave plates (AHPs) is less dependent on wavelength than the standard methods because its principle is based on the geometric phase. ${ }^{5,6)}$ Specifically, the method using AHPs has no spatial-dispersion, unlike the method using a SLM, and its topological-charge (TC) dispersion is much smaller than that by the method using a SPP. An AHP can convert a plane wave into an OV with a TC of $\ell=2$. Furthermore, optical vortices with even-numbered TCs of more than 4 can be generated by cascading AHPs. ${ }^{7,8)}$

Even using the method with AHPs, however, the residual TC dispersion increases with the spectral bandwidth of the light source, since the retardations of the AHPs and/or the other elements used in the OV generator vary with wavelength. The retardation deviation brings about a nonnegligible TC dispersion for some critical applications like astronomical coronagraph. This effect is more apparent with the cascading configuration for generating a high-order OV.

In view of these circumstances, we improve the OV generator using AHPs so that the spurious OV components are selectively eliminated by use of circular-polarization filters. The novel optical configuration allows us to generate almost a pure OV with even-numbered TCs of which bandwidth exceeds $100 \mathrm{~nm}$.

Before describing our novel configurations, we first examine the TC dispersion of the conventional configuration of Fig. 1 for the generation of an $\mathrm{OV}$ with $\ell=4$. The system consists of a polarizer $(\mathrm{P})$, two axially-symmetric half-wave plates $\mathrm{AHP}_{1}$ and $\mathrm{AHP}_{2}$, a quarter-wave plate (QWP), and a half-wave plate (HWP). The solid and broken arrows respectively show fast and slow axes of the waveplates. The combination of $\mathrm{P}$ and QWP is designed to work as a left circular polarization generator (LCPG). Each AHP increases the TC of a left-circularly polarized OV by 2 as well as flipping the handedness of the circular polarization; therefore, the incident plane wave is perfectly converted to an OV with $\ell=4$ if all polarization elements are ideal.

For generating a broadband $\mathrm{OV}$, all the elements used in Fig. 1 should be perfect within the spectral range of the light source. In practice, however, the retardations of the three kinds of waveplates in Fig. 1, namely AHP, QWP, and HWP, vary with wavelength. Their retardation errors cause polarization leakages between orthogonal circular-polarizations at the respective waveplates. The leaked circularly polarized components with opposite handednesses are then converted to spurious OV components with different TCs. Since the retardation errors depend on wavelength, this phenomenon causes the TC dispersion.

As a typical example, we here consider the case where a photonic-crystal AHP and quartz QWP and HWP, all designed at a wavelength of $633 \mathrm{~nm}$, are used with the system of Fig. 1. Typical variations of their retardations are shown in Fig. 2(a). For example, at a wavelangth of $532 \mathrm{~nm}$, AHP, QWP, and HWP respectively have large retardation errors of $\delta_{\mathrm{A}}=50^{\circ}, \delta_{\mathrm{Q}}=20^{\circ}$, and $\delta_{\mathrm{H}}=40^{\circ}$. In this case, the light from the system of Fig. 1 consists of five OV components with different TCs, as shown in the numerically evaluated vortex spectrum of Fig. 2(b). Although the $\ell=4$ component is still dominant even with a large wavelength deviation of $101 \mathrm{~nm}$, considerable energy is shifted to the other OV components with $\ell=2,0,-2$, and -4 . The existence of such fairly large spurious components in the generated OV is not acceptable by several applications that require wider spectral bandwidth, such as astronomical coronagraph and ultra-broadband spectroscopy.

To overcome the above-mentioned dispersion problem in the generation of a broadband OV using the AHPs, we develop alternative configurations that can reduce the spurious OV components due to the retardation errors. Since the spurious OV components in Fig. 2(b) are caused by the polarization leakage at the waveplates in Fig. 1, the elimination

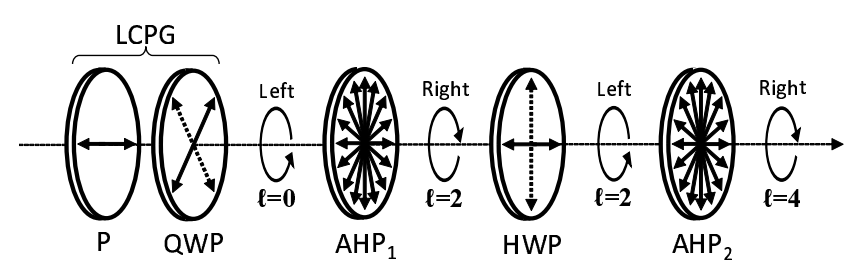

Fig. 1. The original configuration for the generation of an $\mathrm{OV}$ with $\ell=4$ by using two AHPs. 


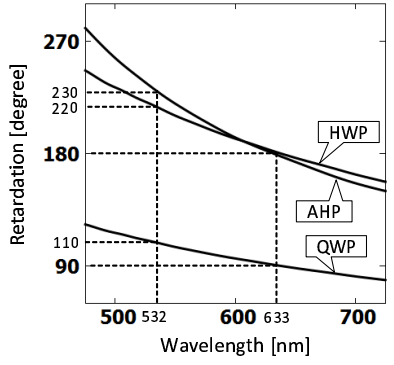

(a) Retardation dispersions

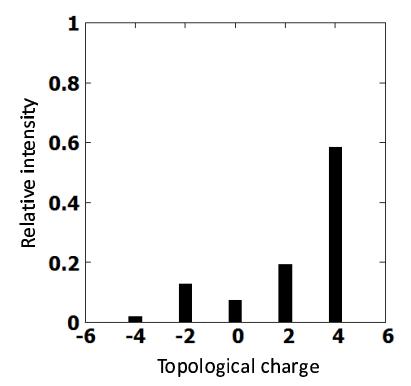

(b) Vortex spectrum
Fig. 2. (a) Typical retardation dispersions of AHP, QWP, and HWP; (b) vortex spectrum from the system of Fig. 1, which is designed for a wavelength of $\lambda=633 \mathrm{~nm}$, for the illumination at a different wavelength of $\lambda=532 \mathrm{~nm}$.

of the unwanted polarization components should allow us to decrease the TC dispersion. On the basis of this idea, we considered two modified configurations illustrated in Figs. 3(a) and (b). In both systems, a right-circular polarization filter RCPF, composed of a QWP and a polarizer, is added behind the system of Fig. 1. This filter reduces the unwanted left circularly polarized component output from $\mathrm{AHP}_{2}$. Moreover, in the system of Fig. 3(b), the central HWP in Fig. 3(a) is replaced with a combination of a RCPF and a LCPG. With the second configuration, the unwanted circularly polarized components are eliminated not only after $\mathrm{AHP}_{2}$ but also between $\mathrm{AHP}_{1}$ and $\mathrm{AHP}_{2}$. Since the difference between Figs. 3(a) and (b) are the number of circular-polarization filters, we will refer to the respective configurations as the single- and double-filter configurations.

To examine the dispersion-reduction capabilities of the novel configurations, we numerically examined the behaviors of vortex spectra of the outputs from the three systems, Figs. 1, 3(a) and 3(b). For this calculation, we here recall the fact that the TC-dispersions of these systems are caused in three steps; (i) the occurrence of retardation errors of waveplates associated with the wavelength variation, (ii) the polarization leakages due to the retardation errors, and (iii) their conversions to spurious OV components. Among them, only

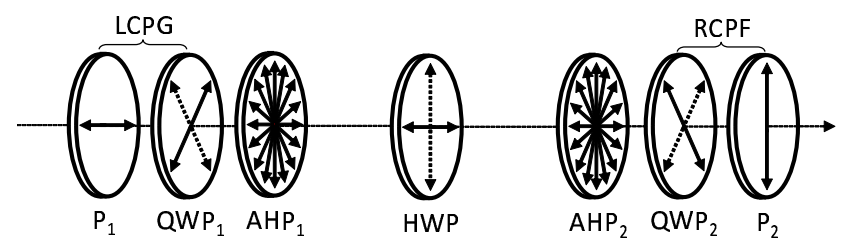

(a) Single-filter configuration

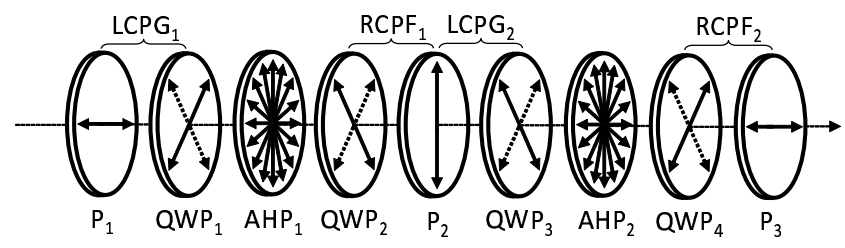

(b) Double-filter configuration

Fig. 3. Modified configurations of the OV generator for reducing the TC dispersion.

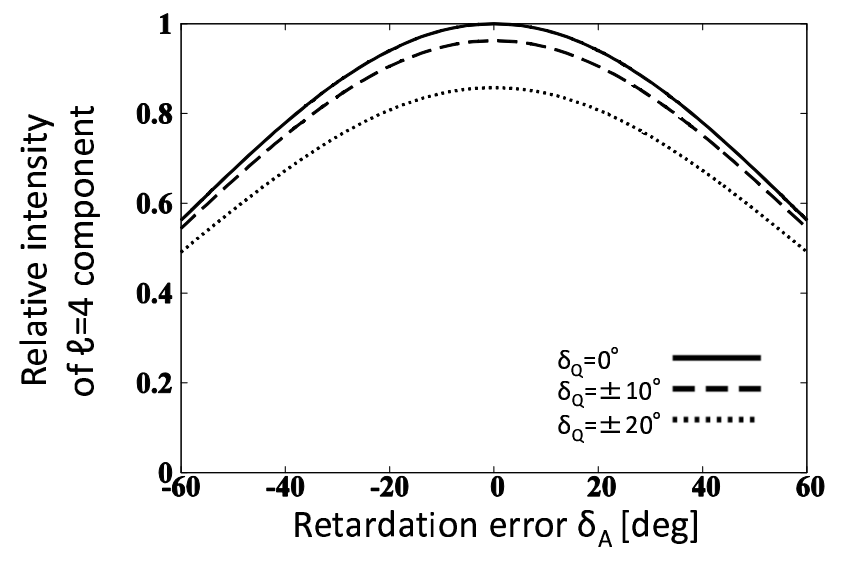

(a) Conventional configuration

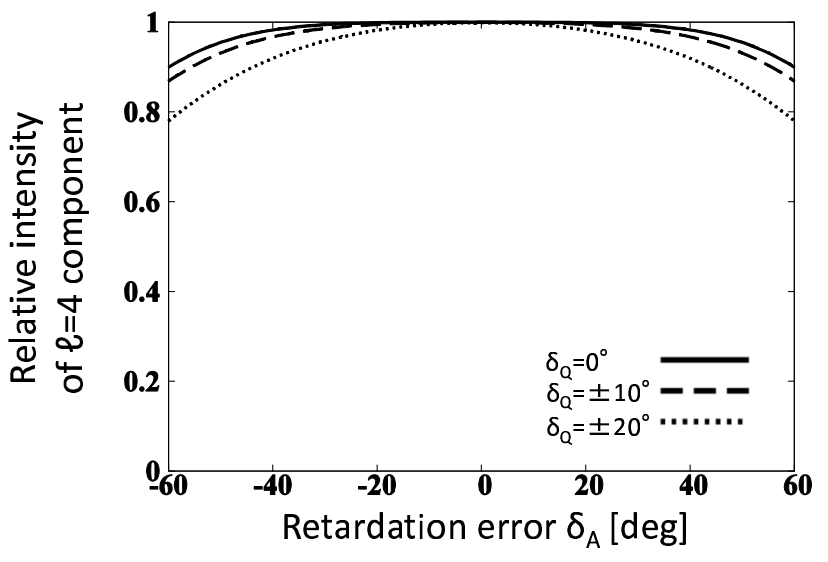

(b) Single-filter configuration

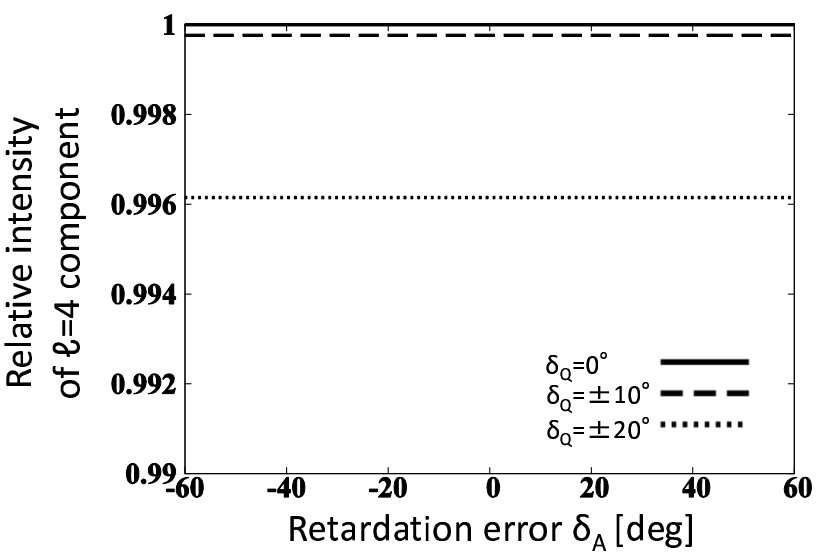

(c) Double-filter configuration

Fig. 4. Relative intensities of the OV component with $\ell=4$, plotted as functions of $\delta_{\mathrm{A}}$ and $\delta_{\mathrm{Q}}$. The vertical axis of (c) is expanded by a factor of 100 .

the first step depends on wavelength as well as materials of waveplates and their design wavelength, as shown in Fig. 2(a). In contrast, the behaviors of the other two steps are determined only by the configuration of the optical system. This implies that the comparison among the three optical systems, Figs. 1,3(a), and 3(b), should be made by the second and third 
steps, namely the relationships between the retardation errors and the spurious OV components, to avoid the uncertainty of the materials of the waveplates and their design wavelength. In view of this fact, we calculated the vortex spectra of the outputs from the three systems for various retardation errors of AHP, QWP, and HWP. In this calculation, we assumed that the QWP and the HWP are made from a same material and that their errors are thereby related as $\delta_{\mathrm{H}}=2 \delta_{\mathrm{Q}}$. Figure 4 shows the relative-intensities of the $\mathrm{OV}$ component with $\ell=4$ in the calculated vortex spectra, plotted as functions of the retardation errors of the AHP and the QWP, $\delta_{\mathrm{A}}$ and $\delta_{\mathrm{Q}}$. In this figure, (a), (b), and (c) are the results of the conventional configuration (Fig. 1), the single-filter configuration (Fig. 3(a)), and the double-filter configuration (Fig. 3(b)), respectively. Note that the vertical axis of (c) is expanded by a factor of 100. Additionally it should be emphasized that the effect of the first step of the TC-dispersion generation can also be considered by combining Fig. 2(a) with this figure. For example, the case at $\lambda=532 \mathrm{~nm}$, discussed with Fig. 2(b), corresponds to $\delta_{\mathrm{A}}=50^{\circ}$ and $\delta_{\mathrm{Q}}=20^{\circ}$ in this figure. As can be understood from Fig. 4(a), the output from the conventional configuration is susceptible to both $\delta_{\mathrm{A}}$ and $\delta_{\mathrm{Q}}$. In contrast, (b) and (c) show that the relative intensities of the $\ell=4$ component are enhanced by use of the modified configurations. This proves the effectiveness of the circular-polarization filters to reduce the spurious OV components. Especially, the double-filter configuration allows us to generate an almost pure OV; over $99.6 \%$ of the generated light has the correct TC. Furthermore, this configuration has a merit that the TC-dispersion is immune to the retardation errors of the AHPs. This implies that the double-filter configuration is suited to generate a broadband $\mathrm{OV}$ with $\ell=4$. We note that the cascading the combinations of a LCPG, an AHP, and a RCPF can also be used for generating a high-order, broadband pure OV.

To show the feasibility of the present principle experimentally, the three configurations were implemented by use of
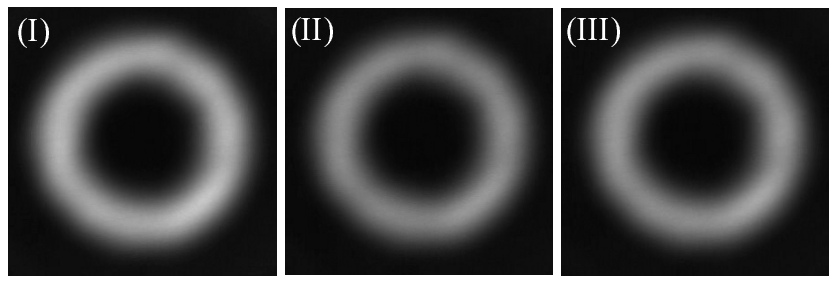

(a) $\lambda=633 \mathrm{~nm}$
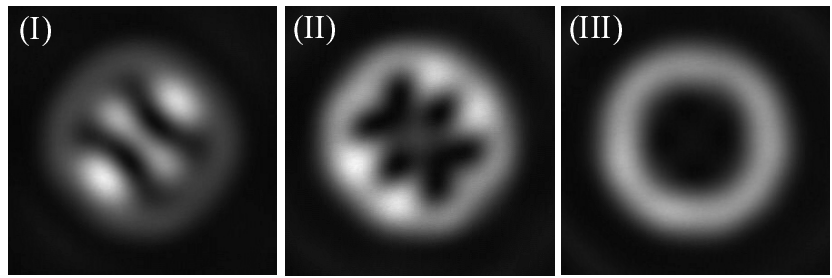

(b) $\lambda=532 \mathrm{~nm}$

Fig. 5. Fraunhofer diffraction patterns of the generated vortices. The upper and lower rows are the results at $\lambda=633 \mathrm{~nm}$ and $532 \mathrm{~nm}$, respectively. (I), (II), (III) are the results by the conventional, single-filter, and double-filter configurations, respectively.

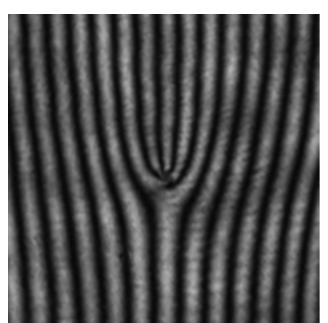

(a) $\lambda=633 \mathrm{~nm}$

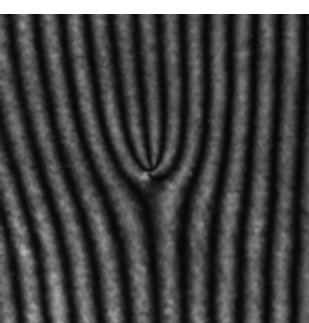

(b) $\lambda=532 \mathrm{~nm}$
Fig. 6. Self-referenced interferograms of the vortex generated by the double-filter configuration (Fig. 3(b)).

photonic-crystal AHPs and quartz QWPs and HWPs, all designed for $\lambda=633 \mathrm{~nm}$. A He-Ne laser and a diode-pumped solid-state laser, respectively operating at $633 \mathrm{~nm}$ and $532 \mathrm{~nm}$, were used with the experiment. We first observed Fraunhofer diffraction patterns of the beams output from the respective configurations as shown in Fig. 5. In this figure, the upper and lower rows are the results at the $\lambda=633 \mathrm{~nm}$ and $532 \mathrm{~nm}$, respectively, and (I), (II), and (III) are the results by the original, single-filter, and double-filter configurations, respectively. At $\lambda=633 \mathrm{~nm}$, all beam profiles are clearly ring-shaped patterns, because all the waveplates are almost ideal. In contrast, at $\lambda=532 \mathrm{~nm}$, whereas the Fraunhofer diffraction patterns of (I) and (II) are considerably distorted owing to the overlapping of the spurious OV components, a clear doughnut-shape profile of (III) free from spurious OV components is obtained even at $\lambda=532 \mathrm{~nm}$ by using the double-filter configuration. Figures 6(a) and (b) are the self-referenced interferograms of the light from the double-filter configuration observed at $\lambda=633 \mathrm{~nm}$ and $532 \mathrm{~nm}$, respectively. Typical interference patterns for an $\mathrm{OV}$ with $\ell=4$, namely the patterns in which a single fringe branches into five fringes, were observed at the both wavelengths. Although we have not quantitatively assessed their vortex spectra yet, these results imply the high purities of the generated OVs. Therefore, this experiment proves the double-filter configuration of Fig. 3(b) is capable of generating an almost pure $\ell=4$ broadband OV, even when considerable retardation errors are present with the AHPs and the QWPs.

In spite of the advantages features, the double-filter configuration has a limitation in the light throughput; the throughput decreases with increases in the retardation errors of the waveplates. This is because the vortex purity is enhanced by eliminating the spurious OV component. For example, the intensity of the output decreased to $58 \%$ from the ideal value in the case where $\delta_{\mathrm{A}}=50^{\circ}$ and $\delta_{\mathrm{Q}}=20^{\circ}$ (shown in Fig. 2(b)). To avoid the throughput deterioration, it is preferable to use waveplates with low dispersions, such as the so-called achromatic waveplates made of two materials. We should note that, an OV with high purity is not obtained from the conventional configuration of Fig. 1 even with the low-dispersion waveplates because of wavelength-dependent residual errors of the waveplates. Especially, it is not easy to make the dispersion of AHP as low as those of achromatic QWP and HWP. Therefore, the double-filter configuration is effective even though in using the low-dispersion waveplates.

In conclusion, we proposed and demonstrated a new 
method to reduce the TC dispersion in an OV generated by using AHPs. The developed double-filter configuration allows us to generate an almost pure OV with $\ell=4$, even when considerable retardation errors are present with AHPs and QWPs. Furthermore, a high-order, broadband pure OV can be generated by cascading the combinations of a LCPG, an AHP, and a RCPF. Our system is applicable to the broadband applications such as ultrafast spectroscopy and astronomical coronagraph.

\section{References}

1) Y. Tokizane, K. Oka, and R. Morita: Opt. Express 18 (2009) 14517.

2) G. Foo, D. M. Palacios, and G. A. Swartzlander, Jr.: Opt. Lett. 30 (2005) 3308.

3) I. Mariyenko, J. Strohaber, and C. Uiterwaal: Opt. Express 13 (2005) 7599.

4) G. A Swartzlander, Jr.: Opt. Lett. 31 (2006) 2042.

5) G. Biener, A. Niv, V. Kleiner, and E. Hasman: Opt. Lett. 27 (2002) 1875.

6) L. Marrucci, C. Manzo, and D. Paparo: Phy. Rev. Lett. 96 (2006) 163905.

7) L. Marrucci, C. Manzo, and D. Paparo: App. Phy. Lett. 88 (2006) 221102.

8) K. Oka, M. Sakamoto, N. Murakami, R. Morita, and N. Baba: presented at SPIE Photonics West, 7950-15, 2011. (Presentation only) 